$\xi=-1$

\title{
Depth-Dependent Optical Stiffness Toward Water-Air Interface
}

\author{
M. S. Mat Yeng ${ }^{1}$, S. K. Ayop ${ }^{1,2 *}$ and I. R. Mustapa ${ }^{1}$ \\ ${ }^{I}$ Department of Physics, Universiti Pendidikan Sultan Idris, Tanjong Malim, Perak, Malaysia. \\ ${ }^{2}$ Research Institute for Electronic Science, Hokkaido University, Sapporo, Japan. \\ *Corresponding authorE-mail:shahrul.kadri@fsmt.upsi.edu.my
}

\begin{abstract}
This research attempted to quantify the optical stiffness of trapped polystyrene bead toward water-air interface. The optical tweezers with $975 \mathrm{~nm}$ wavelength was used to optically trap a single 3 micron diameter of bead in a water-contained cell with air exposed top water surface. The optical stiffness was justified on effective radius $\left(r^{*}\right)$ of the bead lateral spatial distribution. The scattered light signals due to the trapped bead at different laser focus height from the bottom glass-water interface (less than $20 \mu \mathrm{m})$ and laser trapping powers (1.7 $\mathrm{mW}$ to $7.5 \mathrm{~mW}$ ) were analyzed to investigate the $r^{*}$ profile. It was found that within our experimental condition, $r^{*}$ was independent of focus height at fixed power and exponentially decay with respect to laser power at fixed focus height.
\end{abstract}

Keywords: Depth Dependent; Optical Stiffness; Spatial Distribution; Water-Air Interface.

\section{Introduction}

Optical tweezers enable non-invasive and physically non-contact manipulation of small micron sized particle using focused laser. Optical tweezers offer more advantages over mechanical tweezers such as micropipette in handling delicate samples [1]. This ultimate manipulation plays important role in manipulation of biological sample which is fragile and easily get flawed[2]. Optical tweezers have been widely used to study the physical properties of microparticles such as cell, protein, DNA, virus, and others. It also can be used to measure material viscosity, elasticity, stiffness, and others possible applications[3][4].

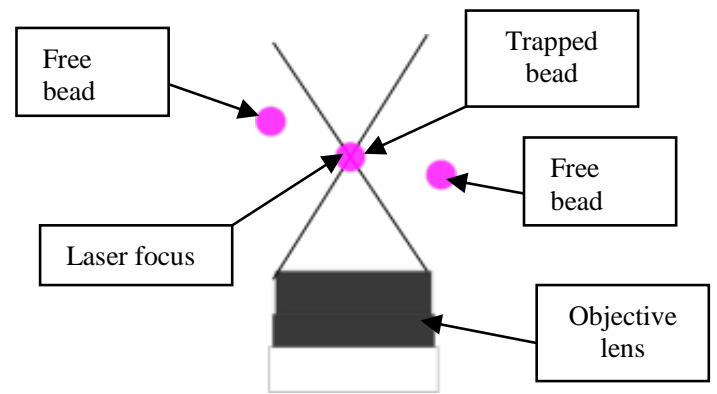

Fig. 1: Optical trapping at the focus of the laser beam

Figure 1 illustrates an optical trap established in an optical tweezers occurs at the focus of the laser beam by an objective lens. The optical tweezers uses a high objective lens to highly focused laser to produce a stable trapped spot[5]. Dielectric particles such as polystyrene or silica beads are attracted to the trap spot due to optical forces. Two main optical forces are responsible for such trapping, namely gradient force and scattering force[6]. The gradient force is due to the intensity gradient of the laser beam. If the used laser beam has Gaussian profile, the intensity of laser is stronger along the propagation axis center and toward the laser focus. The scattering force is due to scattering of incident laser beam that push particle in direction of light propagation. These forces are in balanced in three dimensional space to establish a stable optical trap. Optical stiffness is the measure of how strong an optical trap. High optical stiffness implies strong confinement of the trapped bead. This can be justified through the spatial distribution of the bead. There are several methods to quantify optical stiffness. Among the methods are the escape force method, the drag force method, the equipartition method, the step response method, and the power spectrum method[7]. This reseach employs the equipartition to quantify the optical stiffness. Based on the theorem, the average kinetic energy of particle equals to the average thermal energy of the particle itself as follow

$$
\frac{1}{2} k_{B} T=\frac{1}{2} k<x^{2}>
$$

where $k_{B}$ is Boltzmann constant, $T$ is temperature, $k$ is optical stiffness and $\left\langle x^{2}\right\rangle$ is average standard deviation of bead position along lateral $x$ direction.

Optical trapping applications are commonly performed in liquid environment. The trapping at liquid-air interface is very unique as no study is reported up till now to come up with the exact model to quantify particle trapping at interface level. Previous researchs were more likely to qualitatively decsribe the particle behavior rather than quantify it[8][6][9]. However, this research attempts to trap particle toward the water-air interface. This is very critical to do in order to justify the strength of optical stiffness as it gets close to the free space.

\section{Experimental Setup}

The descriptions of the experiment setup are divided to 4 parts as follow: - 


\subsection{Optical System}

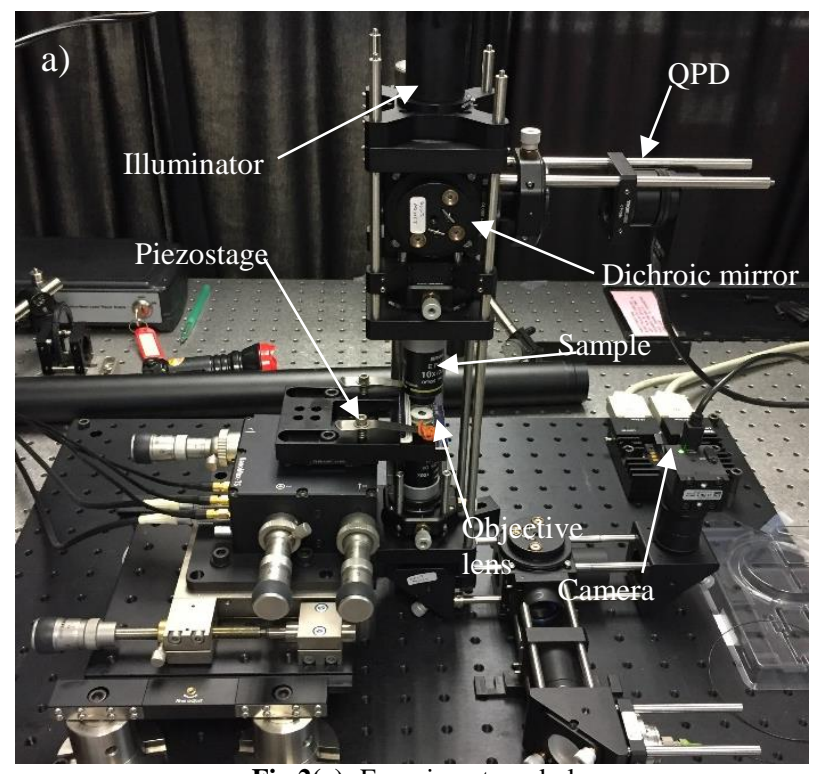

Fig 2(a): Experiment workplace

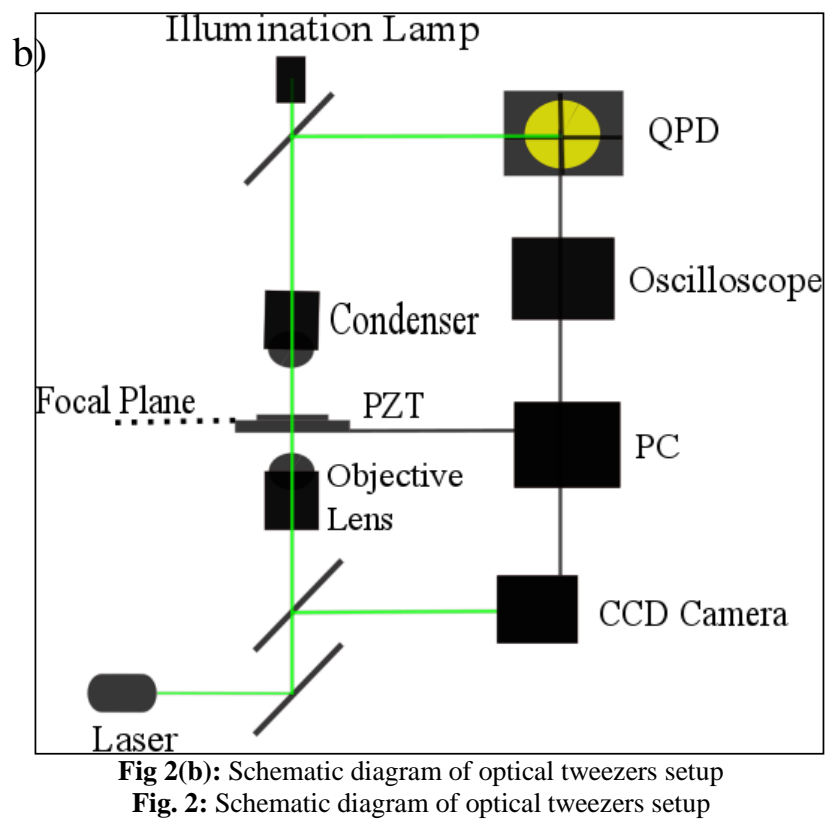

Figure $2 \mathrm{a}$ shows the actual experiment workplace. Figure $2 \mathrm{~b}$ illustrates the schematic diagram of the optical tweezers setup. A $975 \mathrm{~nm}$ laser beam was used as the trapping laser. The laser power was adjusted to be at $1.7 \mathrm{~mW}, 4.6 \mathrm{~mW}, 7.5 \mathrm{~mW}, 10.3 \mathrm{~mW}, 13.2$ $\mathrm{mW}$ and $16.1 \mathrm{~mW}$ as measured at the pupil of the objective lens. The laser was reflected by dichroic mirror and then be tighly focused by the objective lens (immersion oil type 100, 1.23 NA, $0.23 \mathrm{WD})$ toward the sample chamber cell. The image of sample was viewed from CCD camera. The scattered light from the sample was collected by the condenser and then reflected by the dichroic mirror to the quadrant photodiode QPD (PDQ80A, Thorlabs). The QPD sent the signal to oscilloscope and the data was transffered to PC for further analysis. The translation of the sample positioned on a piezostage was controlled by a custommade program on the LabVIEW platform. The height of piezostage was adjusted from $0 \mu \mathrm{m}$ to $20 \mu \mathrm{m}$ along the laser propagation. This corresponds to different apparent height of the bead from the glass-water (bottom) interface.

\subsection{Sample Preparation}

A drop of concentrated polystyrene bead solution $(3.004 \mu \mathrm{m} \pm$ $0.065 \mu \mathrm{m})$ from Polysciences Inc. was diluted in dionized water. Then, the sample was homogenized with electronic shaker. Using micropipette, $1 \mu \mathrm{L}$ of sample was transferred to a custom made sample cell $0.42 \mathrm{~cm}$ radius and $0.018 \mathrm{~cm}$ height producing the water height of approximately $18 \mu \mathrm{m}$. Then the sample was placed on a piezo-controlled microscope stage.

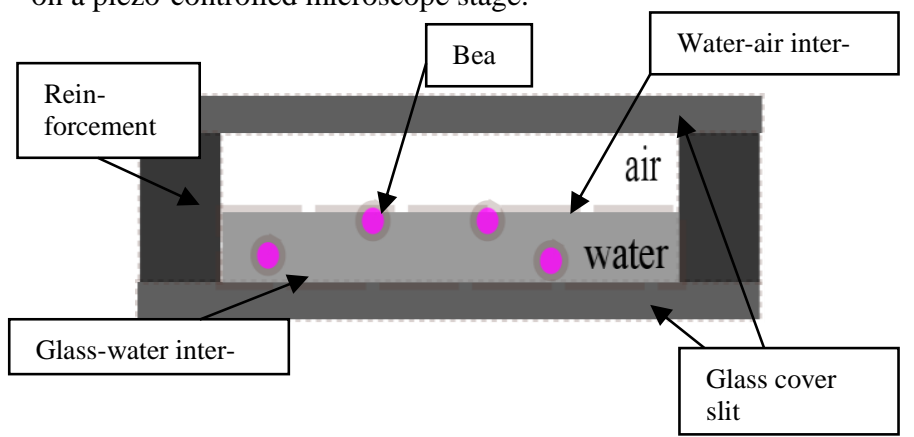

Fig. 3: Sample cell from side view

Figure 3 shows sample cell from side view. The bottom side was glass-water interface and the top side was water-air interface.

\subsection{Optical Tweezers Calibration}

To analyze the motion of the bead, the signal from the QPD must be position-calibrated. The first step is to determine the conversion factor $\beta$ (in $\mathrm{V} / \mathrm{m}$ ) for the QPD diffrential voltage to lateral position. One of the methods for optical tweezers calibration method is the stuck bead method. The stuck bead was scanned across the laser spot in lateral direction at focal plane to obtain QPD diffrential signal versus bead-stage relative distance. Linear fitting across particular distance was performed to obtain $\beta[10]$.

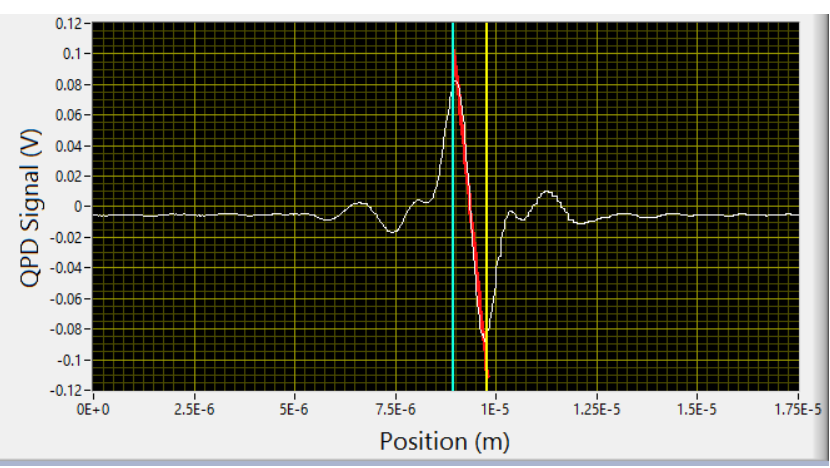

Fig. 4: Graph of QPD signal (V) versus Position $(\mu \mathrm{m})$.

Figure 4 showed a snapshot from our custom-build calibration program of QPD signal $(\mathrm{V})$ versus position $(\mu \mathrm{m})$. The red line represented linear fitting between minimum and maximum points of QPD signal to obtain $\beta$. This calibration was performed for different preset laser power.

\subsection{Effective Radius $\left(r^{*}\right)$ Determination \\ 2.5.}

The spatial distribution of the trapped particle in particular time assembled Gaussian distribution. In this research, spatial contained signal was recorded for $1 \mathrm{~s}$. These data were analyzed in histogram plot and fit using Gaussian function. The effective radius, $r^{*}$ of the distribution was defined as the standard deviation of the Gaussian distribution. $r^{*}$ is directly related to the $\left\langle x^{2}\right\rangle$ which justifies the optical stiffness. Small $r^{*}$ value implies strong optical trap and vice versa. 


\subsection{Height Control}

The bead position was levitated from the bottom interface toward top interface. This is done by changing the laser focus distance from the bottom interface. The spherical abbreviation and refraction might change the position of laser focus[11][12]. Therefore the actual focus distance from the bottom interface $\left(h_{\text {focus }}\right)$ will be less than vertical distance moved the piezostage $\left(h_{\text {piezo }}\right)$. The relation between both distances is as follows

$h_{\text {focus }}=\frac{n_{w}}{n_{g}} \times h_{\text {piezo }}$

where $n_{w}$ refractive index of water and $n_{g}$ glass refractive index.

\section{Results and Discussion}

The effective radius $\left(r^{*}\right)$ was observed and quantified based on the trapping laser power and the focus height from the bottom interface.

\subsection{Lateral Spatial Distribution of Trapped Bead}

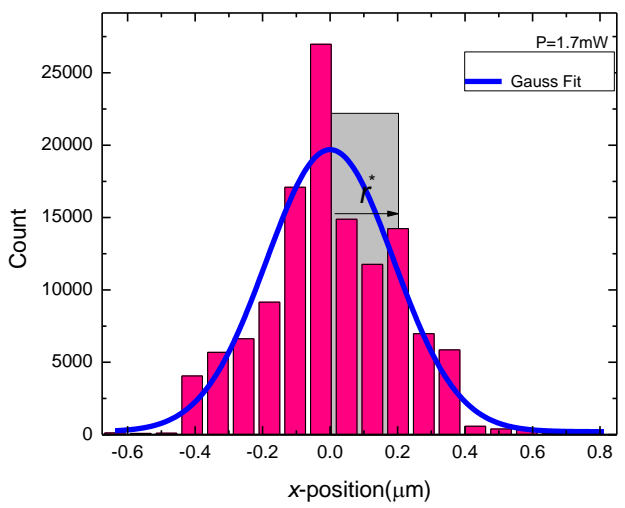

Fig. 5: Spatial distribution along $x$-direction for $\mathrm{P}=1.7 \mathrm{~mW}$ and $h_{\text {focus }}=0$ $\mu \mathrm{m}$

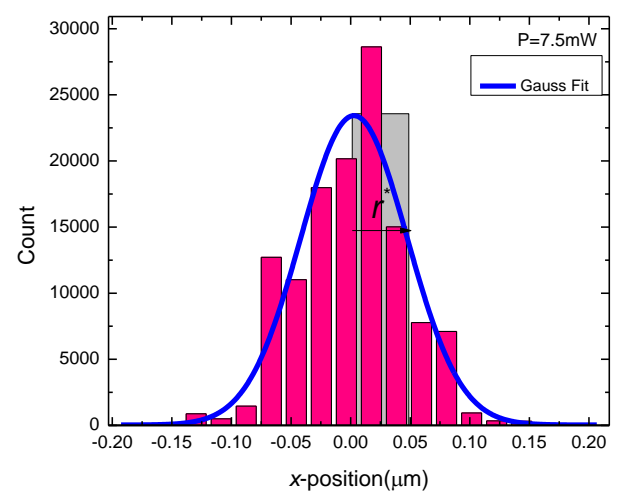

Fig. 6: Spatial distribution along $x$-direction for $\mathrm{P}=7.5 \mathrm{~mW}$ and $h_{\text {focus }}=$ $0 \mu \mathrm{m}$

Figure 5 and Figure 6 show spatial distribution of trapped bead in $x$-direction for the same height, $h_{\text {focus }}=0 \mu \mathrm{m}$ but using two laser powers $1.7 \mathrm{~mW}$ and $7.5 \mathrm{~mW} . r^{*}$ are illustrated in both graph as a grey area where the bead is mostly confined. $r^{*}$ values were obtained by fitting the graphs using Gaussian function. $r^{*}$ is relatively larger in Figure 5 compared to Figure 6. This indicates higher laser power possess higher optical stiffness. One might wonder how can the bead trapped exactly at bottom interface, $h_{\text {focus }}=0$ $\mu \mathrm{m}$. While the laser spot is adjusted to be at the bottom interface, the bead is trap a little off-axial direction since the scattering force is pushing the bead upward[13]. Thus, it is possible to trap the bead near the bottom.

\subsection{Measurement at Constant Power}

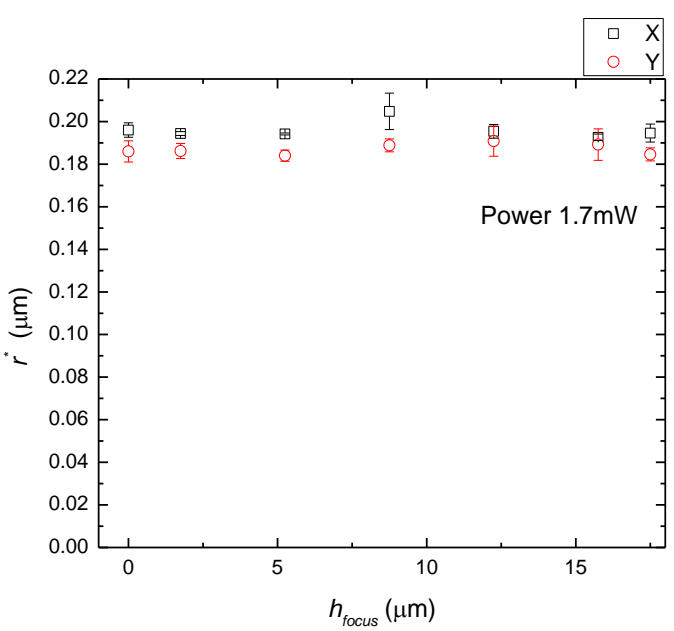

Fig. 7: Graph of effective radius $\left(r^{*}\right)$ versus focus height $(\mu \mathrm{m})$ for $\mathrm{P}=1.7$ $\mathrm{mW}$

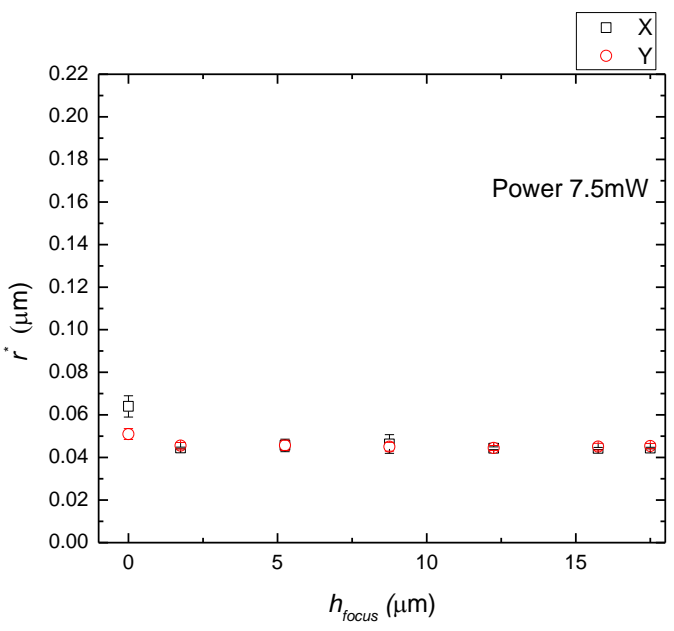

Fig. 8: Graph of effective radius $\left(r^{*}\right)$ versus focus height $(\mu \mathrm{m})$ for $\mathrm{P}=7.5$ $\mathrm{mW}$.

At first, we investigated the dependency of the optical stiffness on the bead height at fixed power. Figure 7 shows that $r^{*}$ is almost constant at any height for fixed $1.7 \mathrm{~mW}$ laser power. The same constant relation also was observed for fixed $7.5 \mathrm{~mW}$ laser power. However, $r^{*}$ for $7.5 \mathrm{~mW}$ laser was generally much smaller than $1.7 \mathrm{~mW}$ for all bead height. This confirmed that the bead was more laterally confined in higher trapping power consistent with the reported result[14].

Figure 9 shows the sequence of trapping at focus height closer to the bottom (a) to the farther from the bottom (f). As the focus height increased, the bead image became blur. This indicated the bead was displaced away that the focal point being pushed away due to dominant scattering force. The pulling axial gradient force was becoming weaker since the intensity of the laser was reduced further from the bottom glass. This finding showed that trapping at position further away from the bottom interface is possible but will trade off with blurring sample imaging. 


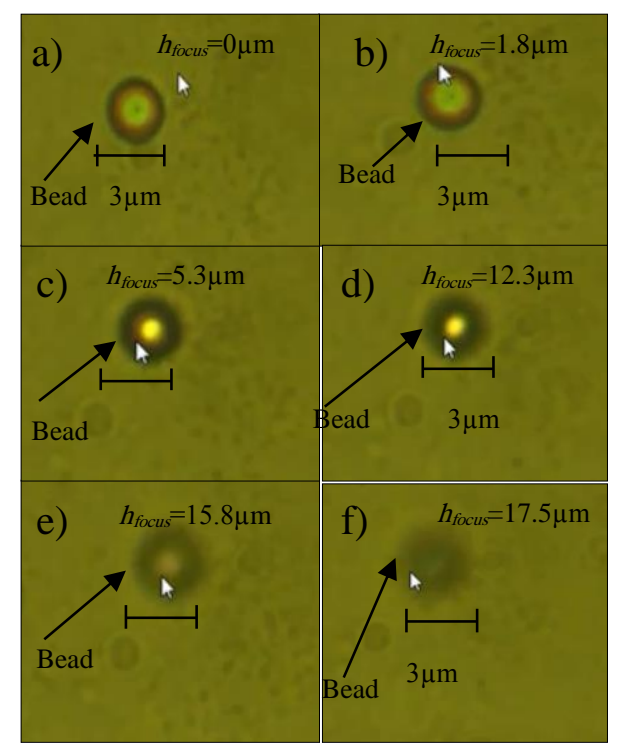

Fig. 9a-f: Sequence of trap bead at various focus height at $1.7 \mathrm{~mW}$ laser power (white arrow indicates the laser spot).

\subsection{Measurement at Constant Height}

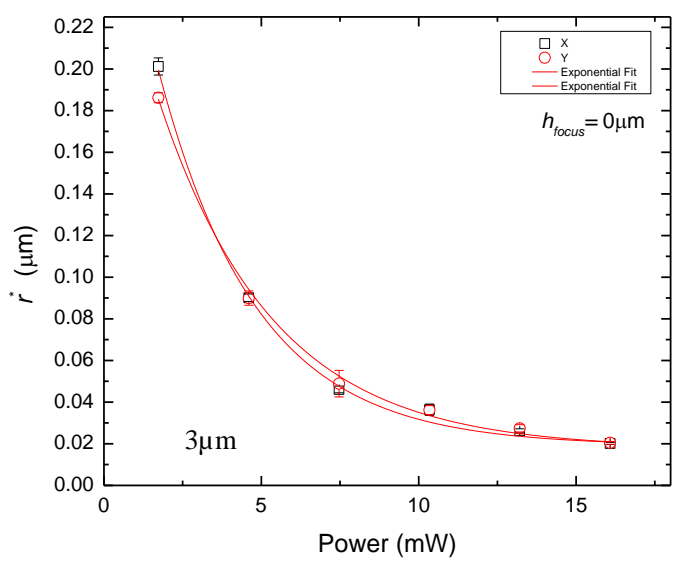

Fig. 10: Graph of effective radius $\left(r^{*}\right)$ versus power (mA).

Subsequently, we investigated the dependency of optical stiffness on laser power at fixed focus height. The Figure 10 shows such result for focus height $0 \mu \mathrm{m}$ (ground). $r^{*}$ decreased exponentially with respect to laser power. This implies that the optical stiffness increased as power increased. We tried to empirically fit the result using $r^{*}=e^{\lambda P}+c$ to determine consistency of $\lambda$ factor.

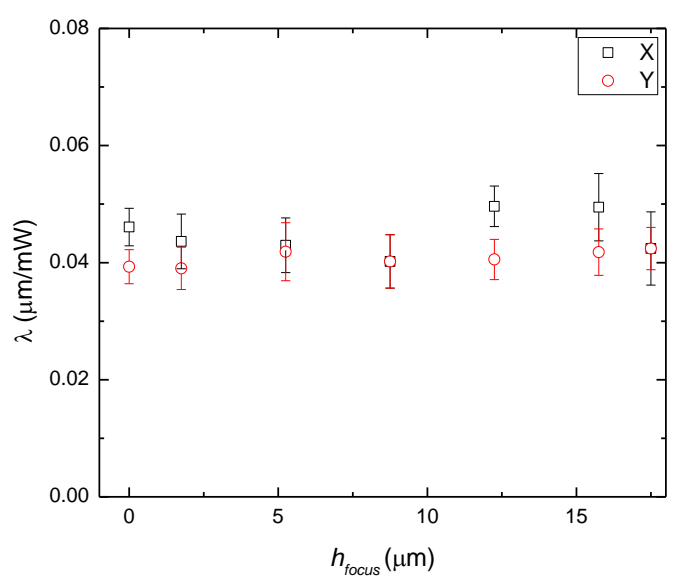

Fig. 11: Graph of decay constant versus focus height $(\mu \mathrm{m})$.
Figure 11 shows the $\lambda$ values at different focus height. Interestingly, the $\lambda$ were almost constant within our range of measurement $\lambda_{x}$ $=0.045 \pm 0.004 \mu \mathrm{m} / \mathrm{mW}$ and $\lambda_{y}=0.041 \pm 0.004 \mu \mathrm{m} / \mathrm{mW}$.

\subsection{Comparison of Effective Radius for Water Medium and Water-Air Interface}

The effective radius was compared for in water medium and at water-air interface as in Table1 below

Table 1: Comparison of effective radius $\left(r^{*}\right)$ for water medium and waterair interface.

\begin{tabular}{|c|c|c|c|c|}
\hline $\begin{array}{c}\text { Trapping } \\
\text { Condition }\end{array}$ & $\mathrm{P}(\mathrm{mW})$ & $\lambda(\mathrm{nm})$ & $r_{x}^{*}(\mu \mathrm{m})$ & $r_{y}^{*}(\mu \mathrm{m})$ \\
\hline $\begin{array}{c}\text { Water-air } \\
\text { interface }\end{array}$ & 1.7 & 975 & 0.19600 & 0.18700 \\
\hline $\begin{array}{c}\text { Water- } \\
\text { medium } \\
{[15]}\end{array}$ & 47.6 & 975 & 0.00549 & 0.00960 \\
\hline $\begin{array}{c}\text { Water- } \\
\text { medium } \\
{[14]}\end{array}$ & 85.3 & 975 & 0.00501 & 0.00518 \\
\hline $\begin{array}{c}\text { Water- } \\
\text { medium } \\
{[16]}\end{array}$ & $\sim 450.0$ & 980 & 0.01600 & 0.01600 \\
\hline
\end{tabular}

Based on the Table $1, r^{*}$ was relatively smaller for optical trapping at water medium rather than $r^{*}$ at water-air interface. The trap is more confined at higher power compared to lower power.

\section{Conclusion}

The effective radius $\left(r^{*}\right)$ was highly dependent with exponentially decay on the power of laser rather than axial focus height from bottom interface. The optical stiffness strength follow $r^{*}$ dependencies. Even though the bead distribution is more confined in the high laser power, it is less stable at large focus distance. This study finding is significant to interface applications where trapping height and laser power in a sample cell is critical.

For the current measurement setup, the bead trapping was possible near water-air interface. However, the bead image is blurring because the bead was trapped off-focus due to dominant scattering force. In the extended work, we are going to reduce the water thickness and varying objective lens to enable particle trapping at water-air interface.

\section{Acknowledgement}

This work is partly funded by Research Acculturation Collaborative Effort (RACE) by Malaysian Ministry of Education (UPSI Code: 2015-0017-102-62). The first author would like to acknowledge scholarship provided by MyBrainSc program.

\section{Reference}

[1] Jiao G \& Zhang R, "Modeling of micropipette aspiration and optical tweezers stretching of erythrocytes with or without Malaria parasite", Theor. Appl. Mech. Lett., Vol. 3, No. 3, (2013), pp. 61-66.

[2] Conteduca D, Dell'Olio F, Ciminelli C, Krauss TF \& Armenise $\mathrm{MN}$, "Design of a high-performance optical tweezer for nanoparticle trapping", Appl. Phys. A Mater. Sci. Process., Vol. 122, No. 4, (2016), pp. 1-6.

[3] Deufel C \& Wang MD, "Detection of forces and displacements along the axial direction in an optical trap," Biophys. J., Vol. 90, No. 2, (2006), pp. 657-667.

[4] Aziz WNSW, Ayop SK \& Riyanto S, "The potential of optical tweezer (OT) for viscoelastivity measurement of nanocellulose solution,” J. Teknol., Vol. 74, No. 8, (2015), pp. 45-48.

[5] Neuman KC \& S. M. Block, "Optical trapping," Rev. Sci. Instrum., Vol. 75, No. 9, (2004), pp. 2787-2809. 
[6] Gutiérrez-Campos RCA, "Optical trapping of particles at the air / water interface for studies in Langmuir monolayers", Rev. Mex. física, Vol. 56, No. 4, (2010), pp. 339-347.

[7] Baek JH, Hwang S \& Lee YG, "Trap stiffness in optical tweezers", Asian Symp. Precis. Eng. Nanotechnol., Vol. 685, (2007), pp. 1100.

[8] Dasgupta R, Ahlawat S \& Gupta PK, "Trapping of micron-sized objects at a liquid-air interface", J. Opt. A Pure Appl. Opt., Vol. 9, No. 8, (2007), pp. S189-S195.

[9] Zhong M, Wang Z \& Li Y, "Laser-accelerated self-assembly of colloidal particles at the water - air interface”, Chinese Opt. Lett., Vol. 15, No. 5, (2017), pp. 1-5.

[10] Hamid MY \& Ayop SK, "LabVIEW-Based Software for Optical Stiffness Determination Using Boltzmann Statistics, Equipartition Theorem and Power Spectral Density Methods", Adv. Sci. Lett., Vol. 4, No. 2, (2011), pp. 400-407.

[11] Vermeulen KC, Wuite GJL, Stienen GJM \& Schmidt CF, "Optical trap stiffness in the presence and absence of spherical aberrations", Appl. Opt., Vol. 45, No. 8, (2006), pp. 1812-1819.

[12] Neuman KC, Abbondanzieri EA \& Block SM, "Measurement of the effective focal shift in an optical trap", Opt. Lett., Vol. 30, No. 11, (2005), pp. 1318.

[13] Mas J, Farré A, Cuadros J, Juvells I \& Carnicer A, "Understanding optical trapping phenomena: A simulation for undergraduates", IEEE Trans. Educ., Vol. 54, No. 1, (2011), pp. 133-140.

[14] Hamid Y, Ayop SK, Wan Aziz WNS \& Munajat Y, "Spatial Distribution of an Optically Trapped Bead in Water", Bul. Opt. 2016, Vol. 2016, No. 2, (2016), pp. 29-36.

[15] Nor W, Wan S, Kadri S, Yunus M \& Munajat Y, "Simple Determination of the Stiffness of an Optical Trap Using Video Microscopy and Particle Tracking," Bul. Opt. 2016, Vol. 1, No. 2, (2016), pp. $1-6$.

[16] Drobczyński D, Du-Szachniewicz S, Symonowicz K, Glogocka K, "Spectral analysis by a video camera in a holographic optical tweezers setup”, Opt. Appl., Vol. 43, No. 4, (2013), pp. 739-746. 